\title{
Severe and extensive enteritis following colectomy for ulcerative colitis
}

\author{
Greg Rosenfeld MD¹, Hugh J Freeman MD¹, Maura Brown MD², Urs P Steinbrecher MD ${ }^{1}$
}

U lcerative colitis is an inflammatory condition usually confined to the colonic mucosa. However, inflammatory changes have been recorded in the small bowel with ulcerative colitis, specifically in ileal pouch mucosa after colectomy (ie, 'pouchitis') or in the terminal ileum with severe pancolitis (ie, 'backwash ileitis'). There have also been rare previous reports of a more diffuse and distinctive form of diffuse and extensive enteritis following total colectomy that responded to steroids or calcineurin inhibitor use (1-3). Rarely, a fatal form of diffuse enteritis following colectomy has been described $(4,5)$. We report a dramatic presentation of severe steroid-resistant panenteritis in a patient three months after total colectomy for severe ulcerative colitis that responded to tacrolimus.

\section{CASE PRESENTATION}

In 2009, a 43-year-old woman was investigated in another hospital for abdominal pain and diarrhea. Upper gastrointestinal endoscopy and biopsy studies of the stomach and small bowel were completely normal; however, colonoscopy with biopsies demonstrated endoscopic and histopathological changes typical of ulcerative pancolitis. Initially, her colitis responded to prednisone and azathioprine. However, over time, she become more refractory despite treatment with mesalamine, infliximab and certolizumab. This led to a colectomy with an ileostomy in September 2011, with reconstructive pouch surgery planned for a later date.

In January 2012, she presented to the emergency department of the Vancouver General Hospital (Vancouver, British Columbia) with crampy, epigastric pain for approximately one month. In the week before presentation, nausea and vomiting also developed along with

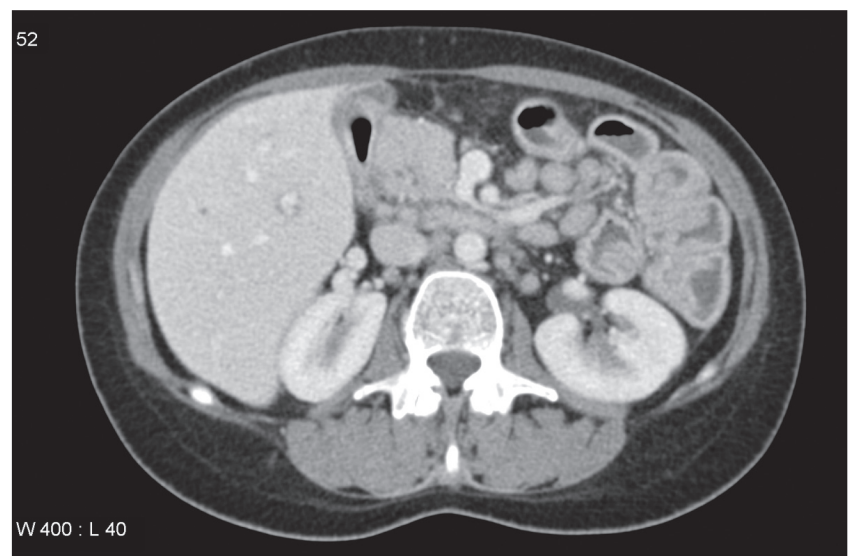

Figure 1) Computed tomography scan showing diffuse bowel edema and mesenteric lymphadenopathy. The bowel wall thickening was seen to extend throughout the small bowel an estimated $2.5 \mathrm{~kg}$ to $5 \mathrm{~kg}$ weight loss. Her bloodwork revealed a mild leukocytosis (white blood cell count $11.4 \times 10^{9} / \mathrm{L}$ ). A computed tomography scan of the abdomen, however, showed diffuse and extensive small bowel thickening (Figure 1). Upper gastrointestinal endoscopy and push enteroscopy showed changes of gastritis and confirmed severe and diffuse enteritis (Figure 2). Biopsies of the duodenum and jejunum documented moderate to severe active inflammatory changes in the small bowel mucosa with cryptitis. There was no intraepithelial
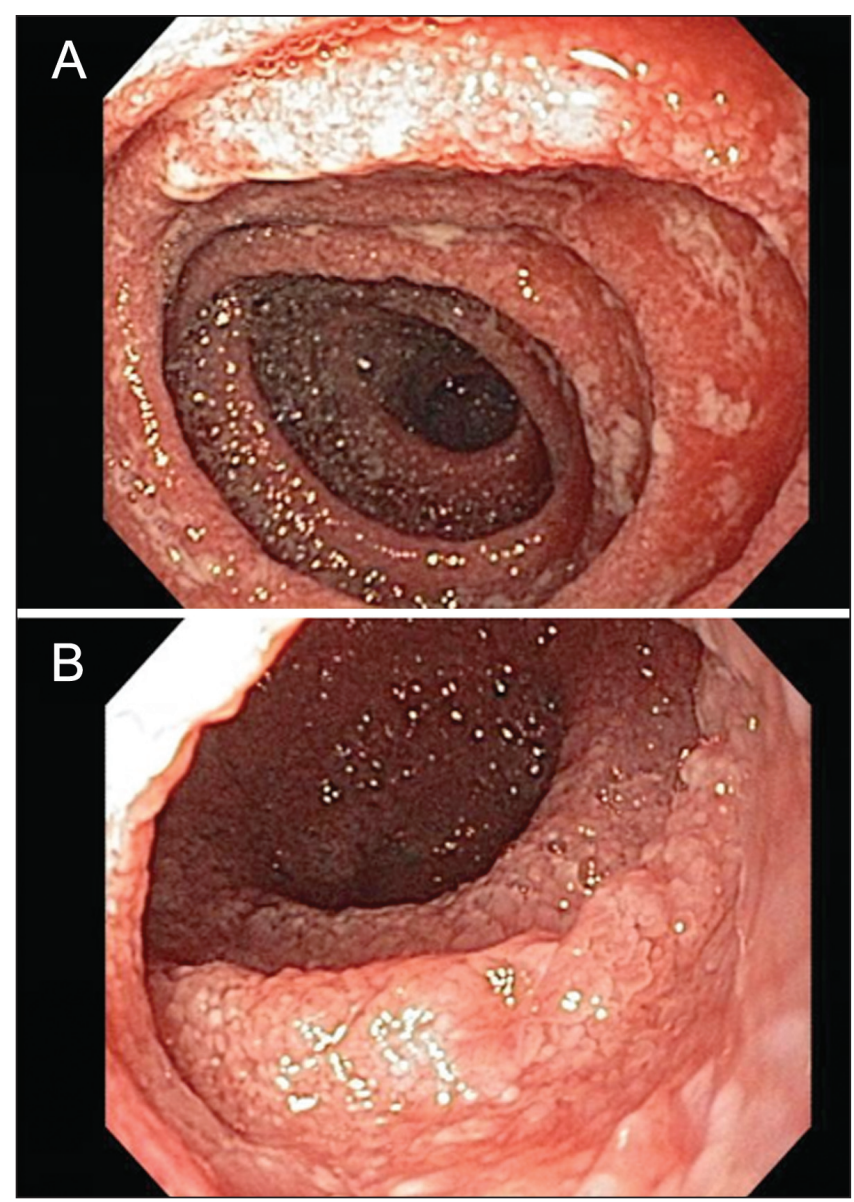

Figure 2) A Jejunum from the morning after admission showing severe edema and inflammation but no discrete ulcerations. B Nineteen days later, after receiving intravenous steroids with some symptomatic improvement but the endoscopic appearance was slightly more severe

${ }^{1}$ Division of Gastroenterology, Faculty of Medicine; ${ }^{2}$ Department of Radiology, University of British Columbia, Vancouver General Hospital, Vancouver, British Columbia

Correspondence: Dr Greg Rosenfeld, Division of Gastroenterology, Faculty of Medicine, University of British Columbia, Vancouver General Hospital,

5th Floor, 2775 Laurel Street, Vancouver, British Columbia V5Z 1M9. Telephone 604-329-9552, e-mail grosenfeld@telus.net

Received for publication June 29, 2012. Accepted July 7, 2012 
lymphocytosis and granulomas were not seen. Fecal cultures and parasite studies were negative, including Clostridium difficile, and antibody levels to tissue transglutaminase were in the normal range. There was no recent history of alcohol, acetylsalicylic acid or other nonsteroidal anti-inflammatory drug use.

Despite treatment with intravenous corticosteroids and parenteral nutrition, symptoms continued and enteroscopy was repeated with biopsies. These showed a persistent and severe diffuse inflammatory process, most severe in the duodenum, with extensive involvement of the visualized jejuno-ileum. Treatment with oral tacrolimus ( $3 \mathrm{mg}$ twice/day) as salvage therapy resulted in improvement with resolution of her pain, permitting complete resumption of normal oral intake and discharge from hospital.

\section{DISCUSSION}

Ulcerative colitis is usually limited to the colon and, occasionally, the distal terminal ileum ('backwash ileitis'). In cases of severe and extensive colitis, total colectomy with ileoanal anastomosis is generally considered to be curative, although pouchitis may develop later in the ileal pouch mucosa. There have also been rare reports of severe and extensive enteritis in patients postcolectomy. In these individuals, the endoscopic and histological findings in the small intestine appear to be more consistent with a diffuse mucosal inflammatory process typical of ulcerative colitis involving the small bowel rather than Crohn disease (1-5). Our patient's presentation was especially impressive because of the documentation of normal endoscopic and histopathological changes before colectomy, and the findings following colectomy that her disease was most prominent in the proximal small bowel along with extension throughout the jejuno-ileum. Furthermore, our patient did

\section{REFERENCES}

1. Valdez R, Appelman HD, Bronner MP, Greenson JK. Diffuse duodenitis associated with ulcerative colitis. Am J Surg Pathol 2000;24:1407-13

2. Rubenstein J, Sherif A, Appelman H, Chey WD. Ulcerative colitis associated enteritis: Is ulcerative colitis always confined to the colon. J Clin Gastroenterol 2004;38:46-51.

3. Corporaal S, Karrenbeld A, van der Linde K, Voskuil JH, Kleibeuker JH, Dijkstra G. Diffuse enteritis after colectomy for ulcerative colitis: Two case reports and review of the literature. Eur J Gastroenterol Hepatol 2009;21:710-5. not appear to respond to oral or intravenous corticosteroids, but required parenteral nutrition and the calcineurin inhibitor tacrolimus.

Other diagnostic considerations may normally include an infectious, ischemic, toxic or immunological cause, but these were excluded. Cultures were negative, including studies for $\mathrm{C}$ difficile, and there was no history of alcohol or nonsteroidal anti-inflammatory drug use. Although celiac disease has occasionally been associated with ulcerative colitis, serological evaluation for tissue transglutaminase antibodies was negative and biopsies failed to show the usual findings of celiac disease, including intraepithelial lymphocytosis. Crohn disease would normally be considered in this setting but the endoscopic and histopathological changes were typical of ulcerative colitis, and pathological changes in biopsies and surgical specimens showed no granulomas.

An additional consideration here may be an entirely novel form of inflammatory disease. Interestingly, although sporadic rare cases from the United States and Europe have been noted after colectomy, several have been reported from Japan (6), raising the possibility that unique genetic or environmental factors play a role. The etiopathogenesis of this severe and diffuse inflammatory process in the small bowel after colectomy is intriguing. A sudden change in inflammatory mediators or inhibitors following cessation of medical treatment of colitis with potent immunosuppressants or biological agents followed by colectomy could have played a role. Alternatively, a 'graft-versus-host'-like immunopathological process has been suggested (3). Clinicians caring for patients with inflammatory bowel disease treated with colectomy and having the expectation that surgical therapy will be curative should be aware of this distinctive and potentially fatal form of small intestinal inflammatory process.

4. Annese V, Caruso N, Bisceglia M, et al. Fatal ulcerative panenteritis following colectomy in a patient with ulcerative colitis. Dig Dis Sci 1999;44:1189-95.

5. Gooding IR, Springall R, Talbot IC, Silk DB. Idiopathic smallintestinal inflammation after colectomy for ulcerative colitis. Clin Gastroenterol Hepatol 2008;6:707-9.

6. Hori K, Ikeuchi H, Nakano H, et al. Gastroduodenitis associated with ulcerative colitis. J Gastroenterol 2008;43:193-201.

The Canadian Journal of Gastroenterology is now considering a limited number of submissions for IMAGE OF THE MONTH. These are based on endoscopic, histological, radiological and/or patient images, which must be anonymous with no identifying features visible. The patient must consent to publication and the consent must be submitted with the manuscript. All manuscripts should be practical and relevant to clinical practice, and not simply a case report of an esoteric condition. The text should be brief, structured as CASE PRESENTATION and DISCUSSION, and not more than 700 words in length. A maximum of three images can be submitted and the number of references should not exceed five. The submission may be edited by our editorial team.

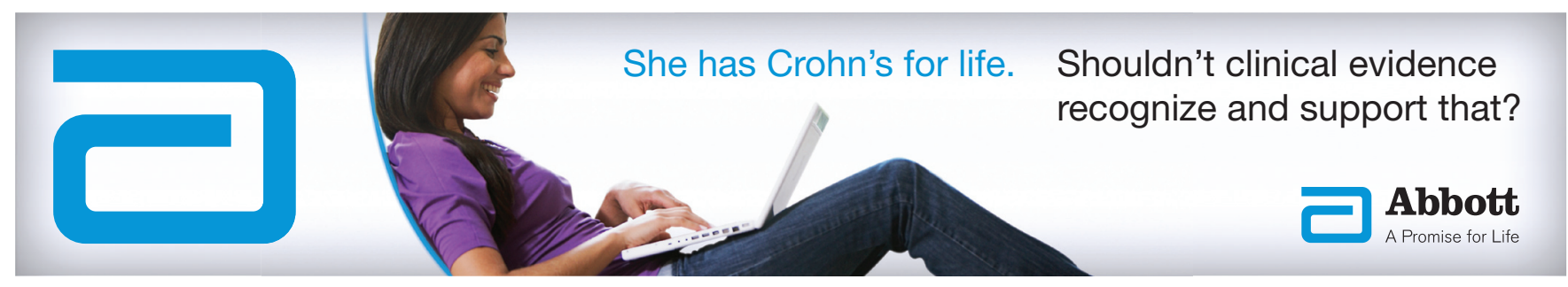




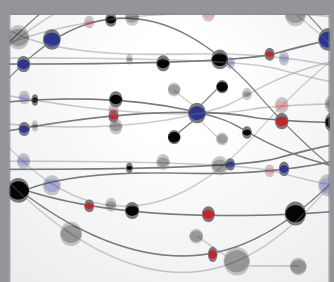

The Scientific World Journal
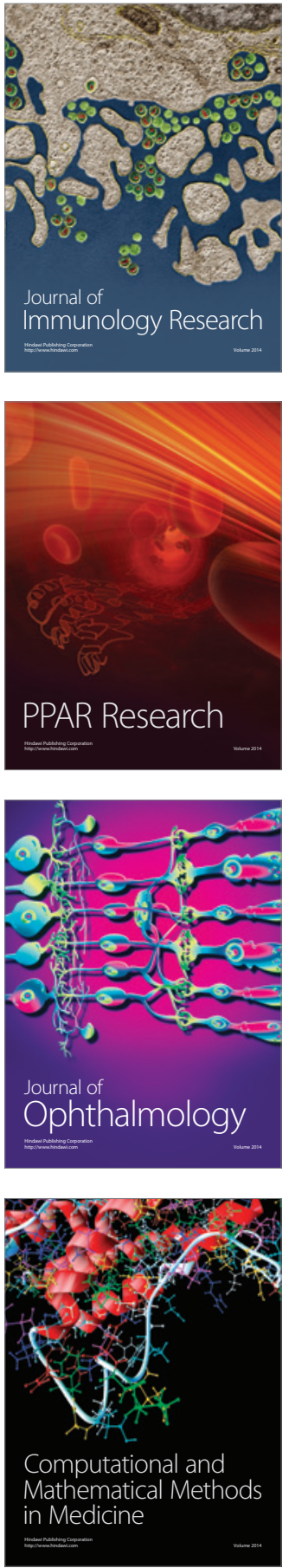

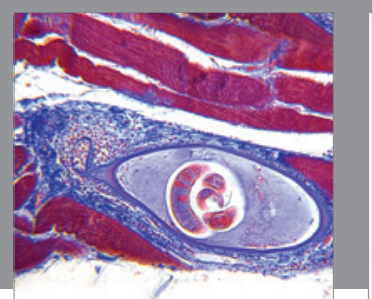

Gastroenterology Research and Practice

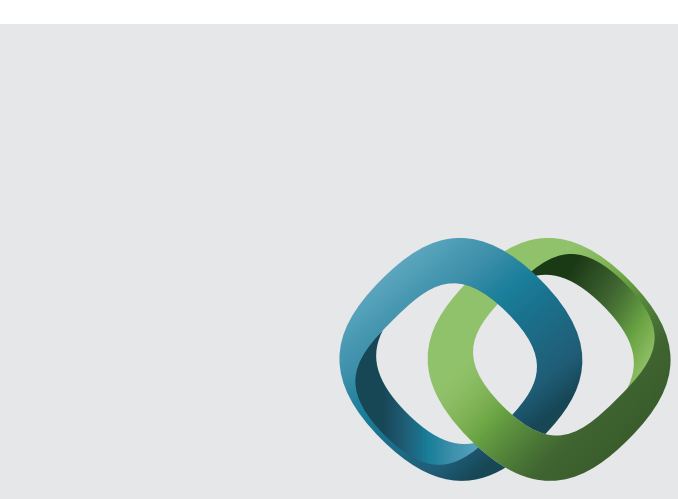

\section{Hindawi}

Submit your manuscripts at

http://www.hindawi.com
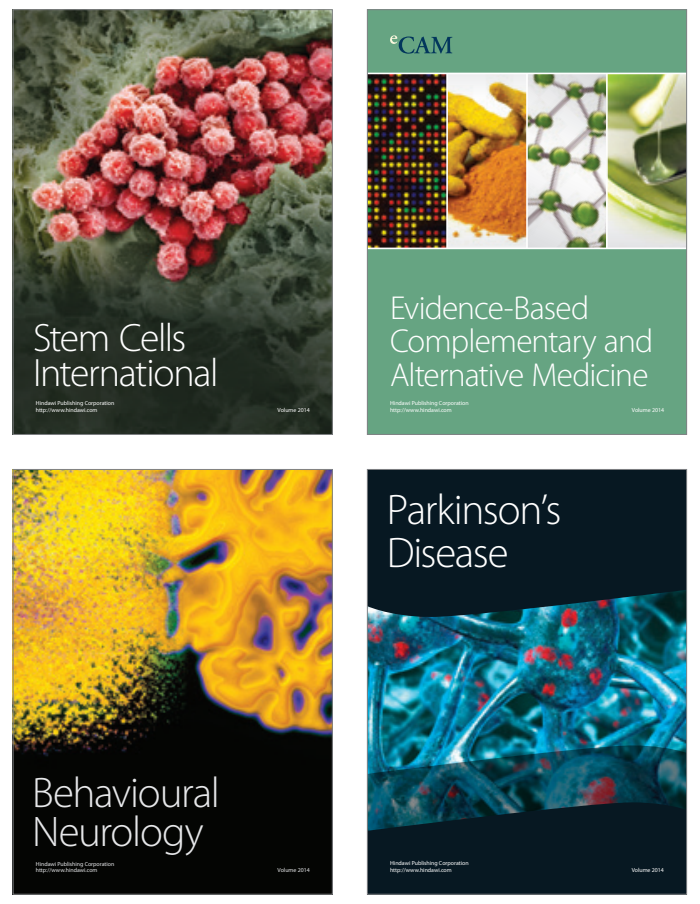
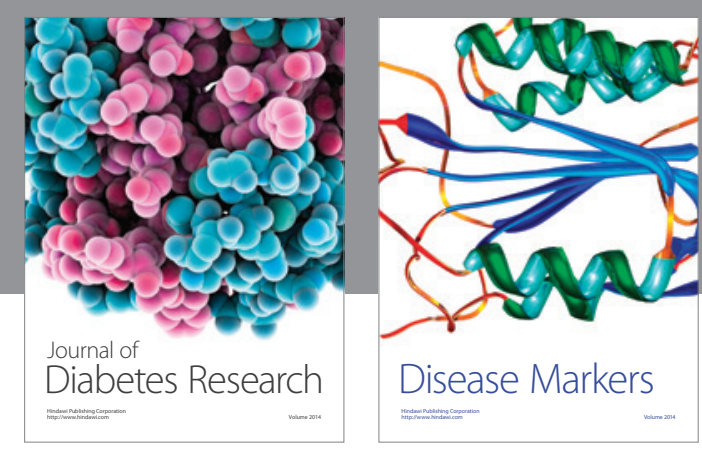

Disease Markers
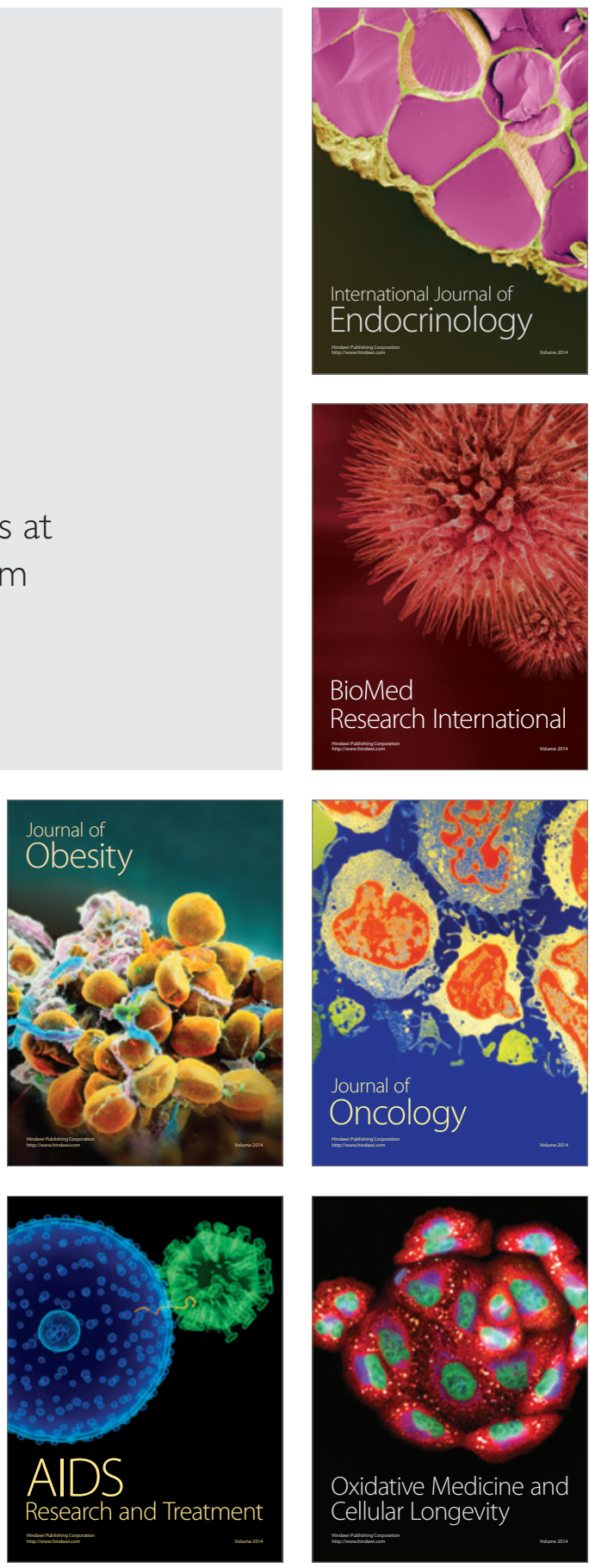